\title{
Masochism in the laboratory rat: An experimental demonstration*
}

\author{
PHILLIP C. GREEN† \\ Bowling Green State University, Bowling Green, Ohio 43403
}

In an attempt to replicate and extend an experimental demonstration by Keller and Schoenfeld concerning transformation of electric shock into a secondary reinforcer, separate groups of rats learned to leverpress for either food or reinforcing electrical stimulation (ESB) to the medial forebrain bundle. The secondary reinforcer was mild footshock, presented through the lever during conditioning. In extinction trials, continued footshock produced greater resistance to extinction than did no shock in both food and ESB groups, suggesting that footshock had assumed the properties of a secondary reinforcer.

A commonly observed effect of punishment is to produce either temporary or permanent suppression of the punished response. Clinical evidence, however, suggests that humans may sometimes actively seek aversive stimulation in the form of mental anguish or even physical pain. Persons willing to suffer such mistreatment for the sake of satisfying an important need (e.g., attention, recognition) may receive some degree of reinforcement from this punishment. In a theoretical sense, punishment becomes rewarding because it is coupled with a primary need such as affection or attention.

Assuming this to be a valid phenomenon in humans, one may ask if an analogue for this behavior exists in lower mammals. An appropriate laboratory demonstration would be one in which an intrinsically aversive stimulus could be given the properties of a secondary reinforcer and serve to bring about a previously learned response to a nonaversive reinforcer.

A number of investigators have demonstrated apparent increased resistance to extinction following aversive stimulation (Gwinn, 1949; Solomon, Kamin, \& Wynne, 1953; Whiteis, 1956; Miller, 1960). In a more recent series of studies (Brown, Martin, \& Morrow, 1964; Brown, 1968; Brown, Beier, \& Lewis, 1971), rats learned to escape from an electrified startbox and runway into a safe goalbox. During extinction, in which the startbox was made safe, Ss forced to cross an electrified runway to reach the safe goalbox showed greater resistance to extinction, in contrast to what would be predicted from reinforcement theory.

* This research was supported, in part, by NSF Departmental Development Grant GU-3175.

tThe author would like to express appreciation to Mr. Thomas Grachek, who helped run some of the $S$ s used in the study.
Keller \& Schoenfeld (1950) cite an analogous paradigm in which rats were trained to leverpress for food. With each response, food was obtained and, simultaneously, mild footshock was administered through the lever. Following performance stabilization, the food dispenser was disconnected and extinction trials began. After some decrease in lever rate, rats again received footshock, but no food, each time a response occurred. The result was a noticeable acceleration in response rate, appearing as a hump in the cumulative response curve. "To an uninformed bystander watching the animal's behavior, it appears that the rats enjoy the shocks and are deliberately punishing themselves [p. 308]."

A common element throughout these studies is increased resistance to extinction as a funetion of continued exposure to aversive stimulation. However, to the author's knowledge, no one has applied this paradigm to electrical stimulation of the brain (ESB), in which the more usual finding is one of rapid extinction following withdrawal of ESB. However, in most of the ESB studies, little emphasis has been placed upon the relationship between secondary reinforcement and resistance to extinction. In the few studies which have specifically treated these variables, both positive (Stein, 1958) and negative (Seward, Uyeda, \& Olds, 1959; Mogenson, 1965) results have been reported.

Since the Mogenson study constituted a failure to replicate Stein's finding of increased resistance to extinction and the Seward et al results have been criticized for lack of relevant data, the issue is still unresolved. In the present study, the design was modified from Keller and Schoenfeld to include comparisons of performance by animals working for food (FM), as well as animals working for ESB.

SUBJECTS AND APPARATUS

Twenty male Sprague-Dawley- derived hooded rats of the LE strain were obtained from Blue Spruce Farms, Inc., Altamont, N.Y. They were approximately 150 days old (mean weight $=450 \mathrm{~g}$ ) at the start of the study. Two modified operant conditioning boxes, each measuring $9 \frac{1}{2}$ in. wide $x 9^{1 / 2}$ in. deep $\times 16$ in. high and equipped with a single LVE rat lever, were used. Each test unit was constructed of Plexiglas and was open at the top so that cranial connections could be made for delivering ESB. Each unit was also fitted with a Foringer pellet dispenser and food receptacle, which could be masked off from $S$ by fastening a plate over the receptacle opening. Hunter timers were used to deliver a 60-cycle 500-msec intracranial pulse train every time the lever was activated. At minimal voltage gain, amperage values were individually adjusted and varied from 20 to $40 \mathrm{~mA}$, depending upon the S's response

\section{PROCEDURE}

Animals first were divided randomly into two groups. At a coin toss, one group was designated the FM group, while the other was called the ESB group. Each group was then further subdivided into lever-shock (STB) and nonshock (NSTB) groups for a subsequent extinction phase.

Surgical implant procedures were performed on all $\mathrm{Ss}$, including those not destined for ESB training, to control for the effects of surgical trauma. Commercially available bipolar electrodes (Plastic Products, Inc., Roanoke, Va.) were stereotaxically implanted under Nembutal anesthesia, with the electrode tips aimed at the largest extent of the medial forebrain bundle (MFB). Coordinates were: posterior bregma, $0.5 \mathrm{~mm}$; lateral, $2 \mathrm{~mm}$; and height, $8.75 \mathrm{~mm}$. Following recovery from surgery, roughly estimated to be the point at which weights were seen to stabilize and ranging from 10 to 16 days, all animals were placed on a 23.5'h food-deprivation schedule for 5 days and then trained in six consecutive 30-min daily sessions to press for standard Noyes $45 \cdot \mathrm{mg}$ sucrose pellets. Following attainment of a shaped lever response, the electrode leads were attached to all animals; Group FM continued working on the same food reward schedule as before. In the case of Group ESB, the pellet dispenser was disconnected, the food receptacle masked off, the stimulation circuit activated, and all Ss in this group now received a brief electrical pulse through the implanted electrodes each time the lever was pressed. As a result of this training procedure, four ESB animals were eliminated from the study. Of this group, two Ss stopped pressing, 


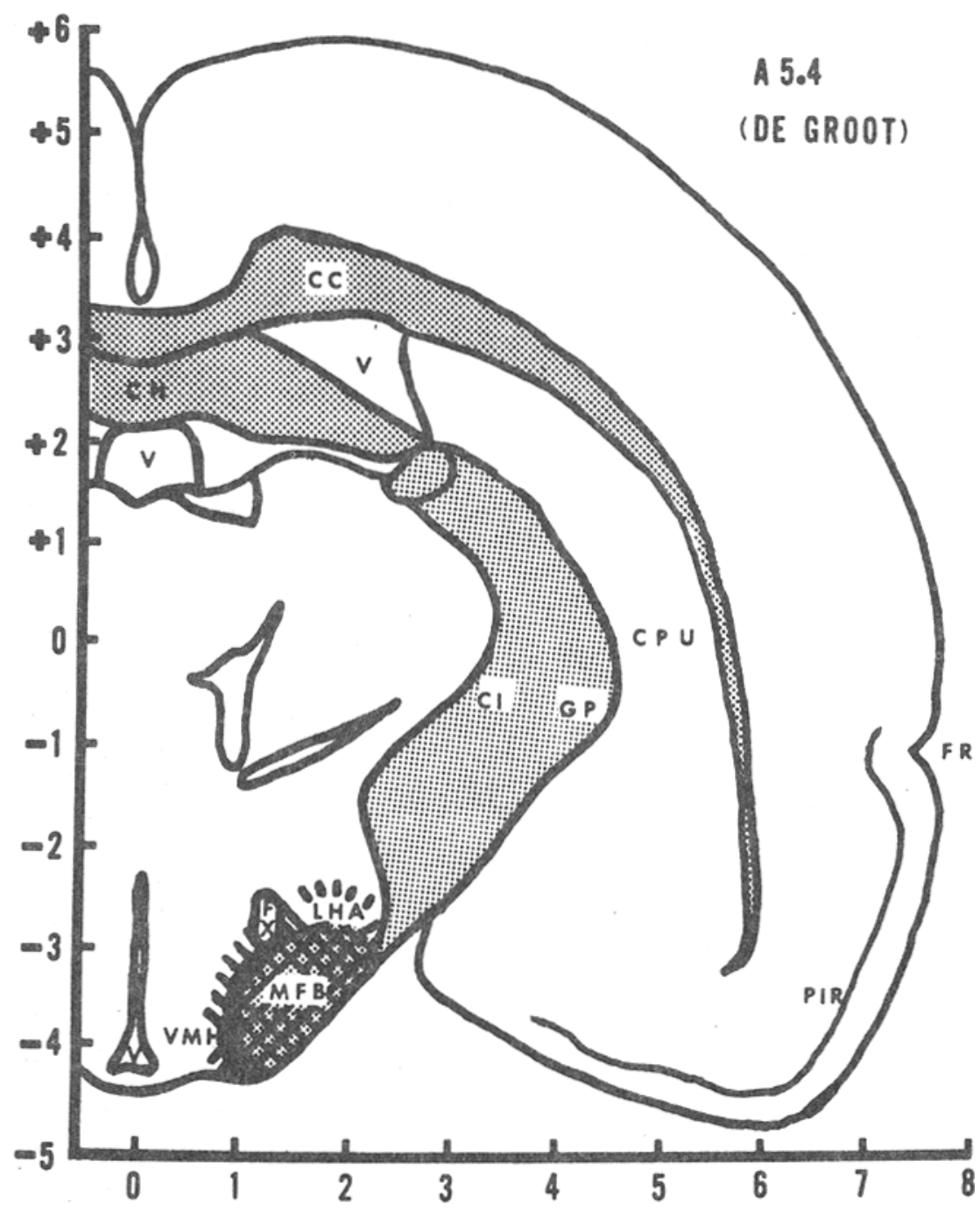

Fig. 1. A tracing of Section A 5.4 (DeGroot Atlas) taken through a large extent of the medial forebrain bundle, showing composite of electrode placements for Es.

apparently because ESB was somehow aversive to them, as judged from postural adjustments observed to be coincident with stimulus onset. The remaining two animals in this group failed to transfer satisfactorily the lever response from food reward to the ESB condition. These animals were replaced with others run concurrently.

The Ss in both FM and ESB groups were given additional training on their respective conditions until relative asymptotic group performance levels had been reached, 24 days later. Following this, for an additional 10-day period, mild shock was delivered through the lever each time contact by $S$ was made during a response. Visual verification that all $\mathrm{Ss}$ could feel lever shock was made in noting the hesitancy with which animals approached the lever immediately after the initial shock fixed in $10 \%$ Formalin solution, embedded in celloidin, carefully blocked at the electrode site, and cut in 70-micron sections. A cresylviolet procedure was used to stain cell bodies. Figure 1 shows a composite of these electrode sites. As can be seen, the tips of all electrodes were closely grouped at the stereometric coordinates previously given for MFB. In the case of the four ESB animals eliminated from the study for failure to leverpress, electrode sites were also analyzed and placements were found to be generally comparable to those of animals successfully completing the study, thus emphasizing the amount of variability characterizing effects of brain stimulation upon behavior within and between species.

\section{RESULTS}

Figure 2 summarizes performance of food $(A, B)$ and ESB-reinforced (C, D) groups, respectively, through the four phases of the study: (1) lever training, (2) acquisition, (3) shock to bar (STB), and (4) extinction. The first phase, in which all animals were trained to press the lever to obtain food reinforcement (Panel 1), showed that acquisition of this response was rapid and relatively uniform, reaching a composite group mean level of 114.25 responses during the final $30-\mathrm{min}$ training session. No statistical differences were measured among treatment groups for the last 2 training days of this phase.

\section{Acquisition}

In this phase (Panel 2), ESB groups $(C, D)$ showed the response spurt characteristically seen in animals working under ESB reinforcement, particularly from Days 5-6 on, showing a pronounced composite elevation from the final 2-day block of lever training to 142.00 responses and then rising to 232.00 on the final test session of this phase. An analysis (Mann-Whitney $U$ test) indicated statistically significant differences in performance $\left(\mathrm{U}=1, \mathrm{~N}_{2}=4, \mathrm{p}=.029\right.$ ) between related $F M$ and ESB groups (A vs $D ; B$ vs $C$ ) on this measure. FM groups $(A, B)$, showing a continuous elevation from the final test block of the lever training phase, established a more gradual acquisition rate, asymptoting at 137.25 in the acquisition series by Day 17 and showing a mean response level of 144.50 by Day 22 .

\section{STB}

In this phase, all animals continued to work under the previous conditions, except that now the lever was electrified such that $S$ received a moderate shock with each lever contact. In both FM and ESB groups, although initial reduction in response frequency was seen, gradual recovery cccurred over time. Composite 
performance for FM Groups A and B (Panel 3) rose from means of 117.00 and 109.00 to 135.00 and 133.00 , respectively, with no statistical differences measured between them. Unexpectedly, however, ESB group means showed significant differences during this test phase, probably reflecting the greater sensitivity to lever shock and slow recovery of one animal in Group D. However, both C and $D$ groups showed signs of recovery by Day 10 of this phase, although they never reestablished the levels attained in Phase 2, reflecting the noxious effect of even mild levels of shock.

\section{Extinction}

This final and critical test (Panel 4) assessed the effect of termination of primary reinforcement for $F M$ and ESB groups, but with lever shock retained for half of each main treatment group (STB). As can be seen from the curves, for the FM groups, the STB subgroup showed significantly greater resistance to extinction than did the NSTB group ( $p=.05$, Mann-Whitney $U$ test). Even more interesting, however, was the performance of the ESB groups in which the same phenomenon occurred, with the STB Ss showing greater resistance to extinction but with group differences falling just short of significance.

DISCUSSION AND CONCLUSIONS

This study successfully replicated the finding of Keller and Schoenfeld and others that rats can be trained to apparently work for punishing reinforcement. In addition, perseveration of a previously ESB-reinforced response was demonstrated. Despite the somewhat tenuous nature of evidence supporting the latter finding, it is nevertheless noteworthy in that, as is well established in the literature concerning behavioral aspects of ESB preparations, extinction can usually be accomplished in several trials and subsequent reinstatement of the response may require some priming (Olds \& Milner, 1954).

It should be noted further that performance comparisons between FM and ESB animals are difficult, for several reasons. First, we still understand incompletely how motivational substrates are tapped by electrical stimulation of the brain and how these states relate to such traditional drive states as thirst or hunger. That there are clear differences in behavioral characteristics of the two types of states cannot be ignored. Further, in the present study, time constants reflecting the two procedures differed considerably. Animals working for sucrose waited approximately 2 sec for the delivery mechanism to deliver a

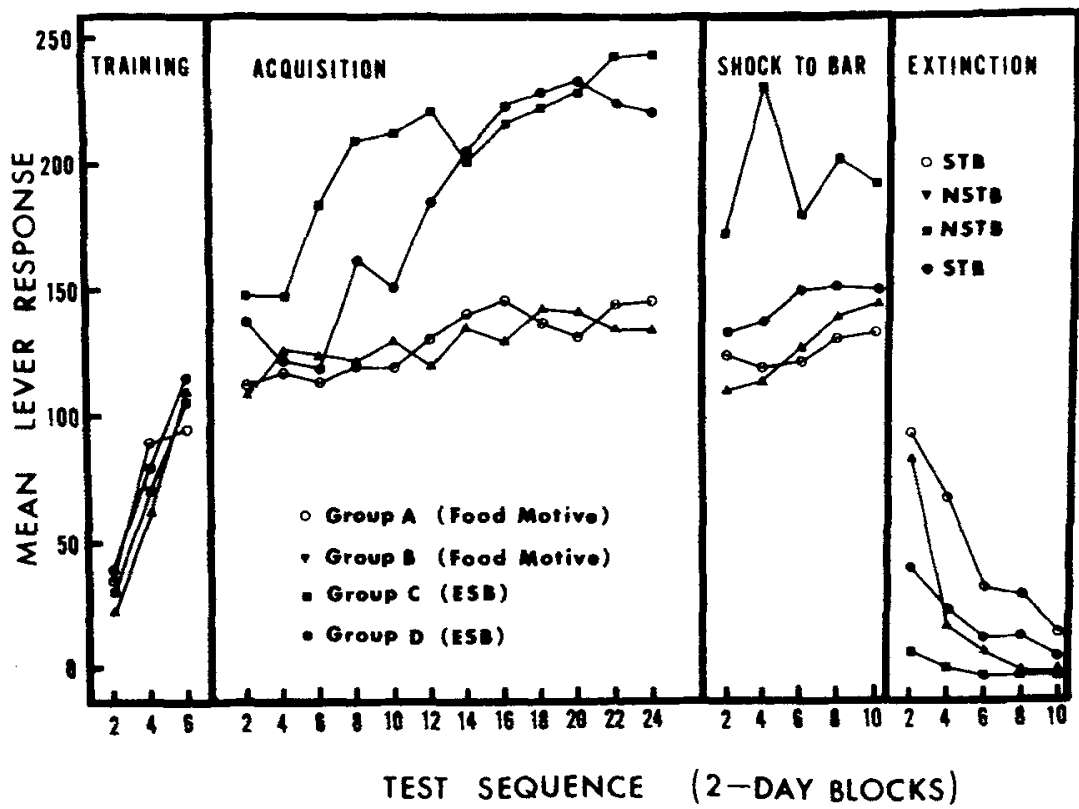

Fig. 2. Summary of performance by FM and ESB groups over the four phases of the study. Each point represents the mean of two consecutive 30 -min test sessions.

pellet to the food receptacle, then spent another 10 to $12 \mathrm{sec}$ obtaining and consuming the pellet before responding to the lever again. By comparison, ESB animals received a single 500-msec electrical pulse train following each response, after which the circuit was instantly reset for subsequent responses. Since the only time-limiting factor was ESB-recovery time, animals in these groups could and did establish higher response levels than FM animals. However, response frequencies for ESB animals were some what below those noted in other studies for lever response frequencies under optimally rewarding stimulation. Although some inter-S variability occurred, response levels were generally below 300 responses $/ 1 / 2-h$ session, as compared to 2,000 responses/h reported by Olds and others. That this apparent discrepancy can be integrated acceptably into the literature is indicated by the observation that response levels have been reported to vary considerably as a function of stimulation, as well as parameters of stimulation (Ursin, Ursin, \& Olds, 1966; Olds \& Olds, 1963; Poschel, 1966).

The significant performance differences found between ESB subgroups in Phase 3 can also be explained by the data. Protocol records by one $O$ (T.G.) for this phase indicated that two animals in Group B had shown occasional response lapses while working in the test unit, invariably accompanied by increase in repetitive overt motor activity, suggesting the possibility of short duration petit mal seizures induced by ESB or possibly potentiated by lever shock. Although this might account for the drop in group performance, such behavior was not observed by the writer (P.G.). It is unlikely that this result had an appreciable effect on the extinction phase, however, since it can be seen that, in the transition from the STB to the extinction condition, in spite of its lower response level in Phase 3 relative to Group C, Group D subsequently showed the greater resistance to extinction.

Finally, there are conflicting accounts concerming the effectiveness of rewarding brain stimulation in producing secondary reinforcement. Stein (1958) showed that when sound was paired with ESB, rats pressed more frequently for tone than no-tone in the absence of ESB. Mogenson (1965), however, failed to replicate this result, and Seward, Uyeda, \& Olds (1959) reported no significant differences in extinction rates when a discriminative light stimulus, previously paired with rewarding brain stimulation, was presented alone during extinction. Since the latter study failed to present data which made it possible to determine whether failure to find differences resulted from $S$ variability or from absence of secondary reinforcement, the results remain ambiguous. The positive results of the present study, therefore, reestablish the possibility that external stimuli, used as secondary reinforcers in an ESB paradigm, produce increased resistance to extinction in a manner 
analogous to animals working under more natural motivating conditions.

\section{REFERENCES}

BROWN, J, S. Factors affecting self-punitive locomotor behavior. In B. Campbell and R. M. Church (Eds.), Punishment and cuersive behavior. New York: Appleton-Century-Crofts, 1968.

BROWN, J. S.. BEIER, E, M. \& LEWIS, R. W. Punishment-zone distinctiveness and self-punitive locomotor behavior in the rat. Journal of Comparative \& Physiological Psychology, 1971, 77, 513-520.

BROWN, J. S., MARTIN, R. G., \& MORROW, M. W. Self-punitive behavior in the rat: Facilitative effects of punishment on resistance to extinction. Journal of Comparative \& Physiological Psy chology, 1964, 57, 127-133.

GWINN, G. T. The effects of punishment on acts motivated by fear. Journal of
Experimental Psychology, 1949,39, 260-269.

KELLER, F. S., \& SCHOENFELD, W. N. Principles of psychology. New York: Appleton-Century-Crofts, 1950.

MILLER, N. E. Learning resistance to pain and fear: Effects of over learning, exposure, and rewarded exposure in context. Journal of Experimental Psychology, 1960, 60, 137-145.

MOGENSON $G$ J An attempt to establish secondary reinforcement with rewarding brain stimulation. Psychological Reports, $1965,16,163-167$.

OLDS, J., \& MILNER, P. Positive reinforcement produced by electrical stimualtion of septal area and other regions of rat brain. Journal of Comparative \& Physiological Psychology, $1954,47,419.427$.

OLDS, M. E., \& OLDS, J Approach-avoidance analysis of rat diencephalon. Journal of Comparative Neurology, 1963, 120, 259-295.

POSCHEL, B. P. Comparison of reinforcing effects yielded by lateral versus medial hypothalamic stimulation. Journal of Comparative \& Physiological Psychology, $1966,61,353-359$.

SEWARD,J. P., UYEDA, A. A., \& OLDS, J Resistance to extinction following cranial self-stimulation. Journal of Comparative \& Physiological Psychology, 1959, 52, 294-299.

SOLOMON, R. L., KAMIN, L. J.. \& WYNNE, L. C. Traumatic avoidance learning: The outcomes of several extinction procedures with dogs. Journal of Abnormal \& Social Psychology, 1953, 48, 291-302.

STEIN, L. Secondary reinforcement established with subcortical stimulation. Science, $1958,127,466-467$.

URSIN, R., URSIN, H., \& OLDS, J. Self-stimulation of hippocampus in rats. Journal of Comparative \& Physiological Psychology, 1966, 61, 353-359.

WHITEIS, U. E. Punishment's influence on fear and avoidance. Harvard Educational Review, 1956, 26, 360-373. 